\title{
Temporal reflectance changes in vegetables
}

Dissing, Bjørn Skovlund; Clemmensen, Line Katrine Harder; Ersbøll, Bjarne Kjær; Løje, Hanne; AdlerNissen, Jens

Published in:

2009 IEEE 12th International Conference on Computer Vision Workshops (ICCV Workshops)

Link to article, DOI:

10.1109/ICCVW.2009.5457516

Publication date:

2009

Document Version

Publisher's PDF, also known as Version of record

Link back to DTU Orbit

Citation (APA):

Dissing, B. S., Clemmensen, L. K. H., Ersbøll, B. K., Løje, H., \& Adler-Nissen, J. (2009). Temporal reflectance changes in vegetables. In 2009 IEEE 12th International Conference on Computer Vision Workshops (ICCV Workshops) (pp. 1917-1922). IEEE. https://doi.org/10.1109/ICCVW.2009.5457516

\section{General rights}

Copyright and moral rights for the publications made accessible in the public portal are retained by the authors and/or other copyright owners and it is a condition of accessing publications that users recognise and abide by the legal requirements associated with these rights.

- Users may download and print one copy of any publication from the public portal for the purpose of private study or research.

- You may not further distribute the material or use it for any profit-making activity or commercial gain

- You may freely distribute the URL identifying the publication in the public portal 


\title{
Temporal reflectance changes in vegetables
}

\author{
Bjørn S. Dissing ${ }^{1}$, Line H. Clemmesen ${ }^{1}$, Hanne Løje ${ }^{2}$, Bjarne K. Ersbøll ${ }^{1}$ and Jens Adler-Nissen ${ }^{2}$ \\ ${ }^{1}$ Department of Informatics and Mathematical Modelling \\ Technical University of Denmark, DK-2800 Lyngby, Denmark \{bdi, Ihc\}@imm.dtu.dk \\ ${ }^{2}$ National Food Institute of Denmark \\ Technical University of Denmark, DK-2800 Lyngby, Denmark \\ $\{$ halo,jadn\}@food.dtu.dk
}

\begin{abstract}
Quality control in the food industry is often performed by measuring various chemical compounds of the food involved. We propose an imaging concept for acquiring high quality multispectral images to evaluate changes of carrots and celeriac over a period of 14 days. Properties originating in the surface chemistry of vegetables may be captured in an integrating sphere illumination which enables the creation of detailed surface chemistry maps with a good combination of spectral and spatial resolutions. Prior to multispectral image recording, the vegetables were prefried and frozen at $-30^{\circ} \mathrm{C}$ for four months. During the 14 days of image recording, the vegetables were kept at $+5^{\circ} \mathrm{C}$ in refrigeration. In this period, surface changes and thereby reflectance properties were very subtle. To describe this small variation we employed advanced statistical techniques to search a large featurespace of variables extracted from the chemistry maps. The resulting components showed a change in both the carrot and celeriac samples. We were able to deduct from the resulting components that oxidation caused the changes over time.
\end{abstract}

\section{Introduction}

Quality assessment of food products is a non trivial task which has been approached in different ways over time. Depending on the food product, different parameters are considered important for the overall quality estimation of the food product. Parameters such as surface color, texture and appearance are very general, and should be assessed in most quality estimation scenarios.

Online quality inspection for food process control is today often done by human expert operators who have many years of experience. However, the trend seems to point towards fast non-invasive inspection methods such as Near Infra Red (NIR) technology for quality inspection in different food process control tasks instead. We propose the use of multispectral imaging in the visible as well as the NIR area of the electromagnetic spectrum to quantify chemical properties of food, and thereby stating its level of quality, instead of human operators and as an alternative to standard NIR measurement methods. By employing imaging instead of point measurements it is possible to gain more spatial information about the process, which makes it possible to assess non-chemical as well as chemical quality features. Nonchemical quality features are evaluations of e.g. piece-size, shape and texture.

In this study, we are specifically investigating the quality loss of meal elements for professionally prepared meals with regards to change in surface color after super-chilling and during thawing at $+5^{\circ} \mathrm{C}$ over a period of 14 days. Meal elements are robust semi-prepared convenience components based typically on meat, fish or vegetables and meant for professional use. The authors have recently shown that pre-fried vegetable meal elements have promising properties with respect to high culinary quality and robustness towards freezing and thawing, thereby potentially solving a major hindrance for the use of heat treated vegetables as meal elements [3]. Super chilling involves a partial freezing of the products, which slows down quality deterioration [4]. In [16] an experiment of celeriac stored in an refrigerated environment was carried out and various parameters were measured using traditional methods. Celeriac and carrots were the subjects of this study, where we measured the reflectance properties using a multispectral imaging device called VideometerLab which will be described in the next section. The pre-fried vegetables were produced by a new process for continuous stir-frying in industrial scale, which has been introduced for producing convenience high-quality vegetables [1]. The pre-fried vegetables have a low fat content (typically $1 \%-2 \%$ of the product weight), a texture and flavor similar to what can be achieved in the kitchen, and vitamins are preserved almost 100\% [5]. In subsequent studies it was observed that the products may be frozen and re- 
heated on a frying pan or in a convection oven without any exudation of excess water, which is a major advantage over existing quick-frozen vegetables [2].

\section{Materials and methods}

In the following, the experimental design, the acquisition of digital images of the vegetables and further postprocessing of these are described.

\subsection{Experimental setup}

In the present work the quality of pre-fried vegetables (celeriac and carrots shaped as cubes of size approximately $0.5 \mathrm{~cm}^{3}$ ) were evaluated (e.g. by means of change in color surface) after freezing and thawing. In a pilot plant, the raw products were pre-fried using a special frying machine "the continuous wok" [1]. After frying, the products were packed in $500 \mathrm{~g}$ portions in plastic bags and frozen at $-30^{\circ} \mathrm{C}$. After four months of freezing, the bags with the pre-fried vegetables were removed from the freezer and thawed up to 14 days at $+5^{\circ} \mathrm{C}$ in refrigeration. On each day of analysis (day 2, 4, 8, 10, 12 and 14) one plastic bag was taken out from the refrigerator and the contained vegetables were digitized. For both types of vegetables, the samples were digitized using two petri dishes to create a test and training set. The multispectral images were segmented in two steps, first isolating all vegetable-piece in the image, and then separating the pieces from each other. After segmentation, ratios were calculated for all combinations of wavelengths to remove shadow effects and possibly get better baseline separation in different spectral bands. For each ratio in each vegetable-piece, the 1st, 5th, 10th, 25th, 50th, 75th, 90th, 95th and 99th percentiles were calculated. This yielded a total of 3249 variables, in a test and training set having 193 and 192 observations respectively for the carrot data. For the celeriac data, similar datasets were created yielding a total of 3249 variables with 207 and 206 observations in the test and training set respectively. Obviously we need a way to figure out which ratios best describe changes over time. For this task a penalized LS algorithm called LARS-EN which is described later was employed to find a set of optimal components. Subsequently statistical tests were performed to evaluate if the identified changes were significant.

\subsection{VideometerLab}

The acquisition of data was done using VideometerLab(http://www.videometer.com) which acquires multi-spectral images in up to 20 different wavelengths ranging from 430 to $970 \mathrm{~nm}$. The camera setup is seen in Figure 1a. The object, in this paper, vegetables, is placed inside an integrating or Ulbricht sphere which has its interior coated to obtain high diffuse reflectivity for optimal light conditions. In the top of the sphere a camera is located with the sensitivity spectrum seen in Figure 1c. The sensitivity decays towards the near-infrared area, which means that the illuminating diodes in this area needs more power to achieve the same level of intensity as the visible bands. The LEDs, having the spectral radiant power distributions seen in Figure 1b, are strobing successively, resulting in an image for each LED of dimensionality 1280x960. These are calibrated radiometrically as well as geometrically to obtain the optimal dynamic range for each LED as well as to minimize distortions in the lens and thereby pixel-correspondence across the spectral bands. The well defined and diffuse illumination of the optically closed scene aims to avoid shadows and specular reflections. Furthermore, the system has been developed to guarantee the reproducibility of the collected images. This allows for comparative studies of time series of images [8].

\subsection{Segmentation of the images}

In the experiment we considered one vegetable-piece as an observation. In order to extract each vegetable-piece of the multispectral image seen in Figure 2, a relative difficult segmentation problem is at hand. This is caused by the fact that the individual pieces were not placed in a systematic manner where they were isolated, but instead lie in lumps, touching each other. This means that they cast shadows on each other as seen in Figure 2. Furthermore the pieces have very similar spectral fingerprints which means they cannot be discriminated using purely spectral values.

As an initial step, the background, meaning everything but the vegetables was isolated. This was done using Otsu's method [13] on the multispectral image, projected onto a hyperplane. The projection function used to carry out this projection, optimally separates pixels coming from one of two populations. These populations are described by two types of labels which were manually annotated. One set of labels contained spectra of petri dish and general background, while the other of vegetable surface, either carrot or celeriac. The labeled data was used to calculate the projection function by means of a Canonical Discriminant Analysis (CDA) [11].

In order to isolate each piece, spatial information is needed, especially gradient information. This information acts as a good guide for the segmentation, and together with morphological transformations employed under a markercontrolled Watershed segmentation as described in [9] we were able to do an automatic segmentation of the vegetables. The result of the segmentation is seen in Figure 2. This seems to be a relatively good result, although flaws are present. In the upper left corner of the petri dish, two pieces are merged together as a result of bad gradient information. In the middle right side it is seen how a dim piece has been 


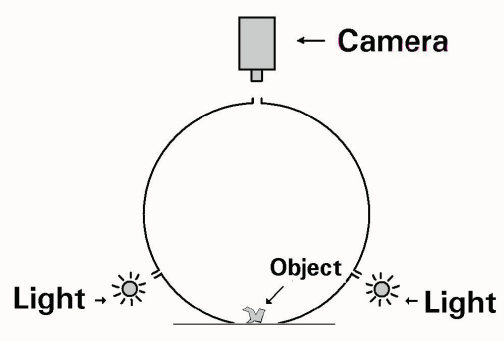

(a)

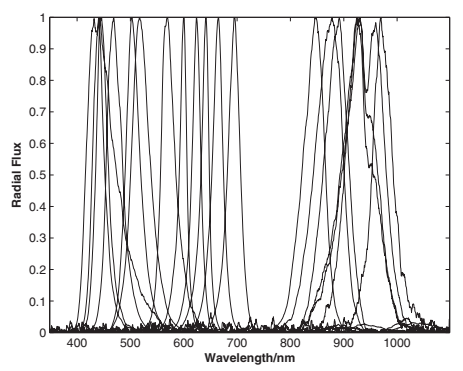

(b)

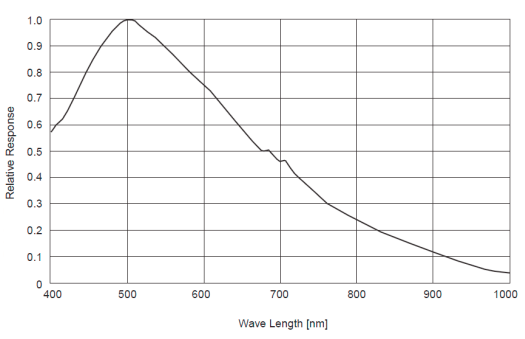

(c)

Figure 1. a) Principle of imaging with integrating (Ulbricht) sphere illumination. The LEDs located in the rim of the sphere ensures narrowband illumination. b) Normalized spectral power distributions of the LEDs located in the VideometerLab. c) Spectral sensitivity of the camera mounted in VideometerLab. It is seen in a) that the camera is placed above the object of interest.

totally ignored by Otsu's method due to its dark appearance. These flaws might be avoided by using an alternative segmentation technique but they were not crucial for the task at hand.

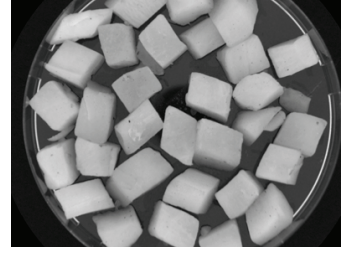

(a)

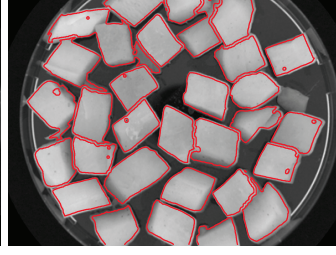

(b)
Figure 2. Both figures show band 10, corresponding to $645 \mathrm{~nm}$ of the carrot sample, on day 2. a) is the pure image and b) has the segmentation result superimposed on the image.

\subsection{Feature selection method}

Having a feature space with $n$ observations and $p$ variables, there are different ways of using this space to describe a variable depending on it. A common technique used to relate the dependent variable and the feature space in a well-posed problem is by using Ordinary Least Square (OLS). Here we have chosen the dependent variable to be the number of the day the observation belongs to, while the independent variables as mentioned earlier are the ratios of the recorded spectrum.

If a problem is well-posed it means among other things that the solution of the problem is unique. Some problems are however not well-posed, which is why many have looked into solving so called ill-posed problems [10] where the covariate matrix does not have full rank. This will always be the case when there are more variables than observations $(p>n)$. If such a problem is to be solved properly using Least Squares (LS), some sort of regularization is necessary. Typically this involves including additional assumptions, such as smoothness of the solution. Tikhonov regularization [15] also known as ridge regression [12] is one of the most common ways of regularizing a linear ill-posed problem or an overdetermined system. The ridge regression minimizes the residual sum of squares like an OLS, but in addition it penalizes the L2-norm of the model coefficients. This means all variables are kept in the model but in a smoothed manner. However, in some situations where $p>>n$, ridge regression is not well suited because it creates very complex and thus very little interpretable models. This also means that if some variables contain none or little information regarding the dependent variable, they will still contribute to the final model and thus induce noise. Another approach to solve $p>>n$ problems is by using subset selection or stepwise selection. These methods choose variables having largest partial correlation with the dependent variable, and discards the rest. This type of model is also sometimes known as a parsimonious model and is often much more interpretable, although unfortunately often yields lesser prediction ability.

The Least Absolute Shrinkage and Selection Operator (LASSO), proposed by Tibshirani in [14] was created to solve this problem. Here an L1-norm penalization of the coefficients is used instead of the L2-norm. This means that a sparse solution, as is the case with stepwise selection, is obtained while still continuously smoothing the coefficients to some degree for good prediction. This approach proved to be an improvement of the ridge regression in many cases, while boosting regression and forward stagewise regression both were invented as alternative methods approximately thereafter. These are all described in [11].

A method able to obtain the solution of all these methods in a computationally fast manner is the Least Angle Regression (LARS)[7], proposed by Efron. This regression method gives rise to at most the same amount of calculations as an ordinary LS. An alternative regularization and variable selection method is the elastic net (EN) by Zou [17], which often outperforms forward stagewise regression as well as lasso regression. The elastic net can be incorporated into the LARS regression, commonly known as 
LARS-EN, and penalizes the L1 as well as the L2 norm of the coefficients; see Equation (1).

$$
\begin{aligned}
L(x, \theta) & =\sum_{i=1}^{n}\left(\sum_{j=1}^{p}\left(\theta_{j} x_{i j}\right)-y_{i}\right)^{2} \\
& \text { s.t. } \sum_{i=1}^{p} \theta^{2} \leq s_{1} \text { and } \sum_{i=1}^{p}|\theta| \leq s_{2}
\end{aligned}
$$

$L$ denotes the loss function, which is the residual sum of squares. $\theta$ are the model coefficients and $\mathrm{y}$ is the dependent variable, in this case the experimental days. $s_{1}$ and $s_{2}$ are the constraint bounds on the LASSO and ridge constraints respectively, which together gives the Elastic Net constraint.

The Contours as well as constraints of a simple 2 dimensional problem, simulated as an example of Equation 1 is also seen graphically in Figure 3. This regression scheme

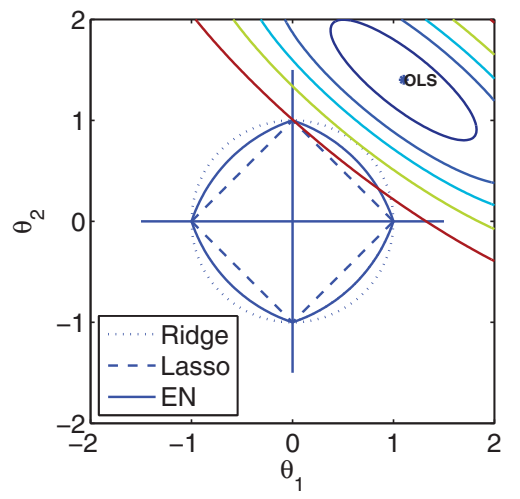

Figure 3. Contours of the residual sum of squares function with the Ordinary LS solution defined as the minimum. The ridge, LASSO and Elastic Net constraints are similarly illustrated. Where the respective constraints and contours intersect, the ridge, LASSO and Elastic Net solutions will be defined.

is especially suited to solve $p>>n$ problems due to the stability of the L2 norm, and the sparsity property of the L1 norm, which is also shown in [6]. Here the LARS-EN efficiently manages to select a set of suiting variables to detect water in different types of sand, which also is the reason why we have chosen to use it to solve the problem in this paper.

\section{Results and discussion}

In order to generalize the model as much as possible a leave one out cross validation (LOO-CV)[11] was used on a training set (A) to estimate the model, and a separate test set (B) was used to evaluate the performance of the model. To further check the repeatability of the model, the training and test set were switched and a new model estimated. Predictions of the estimated models are seen in Figure 4. The boxes in the figures are standard type boxplots and have lines at the 25th, 50th and 75th percentiles. The whiskers are lines extending from each end of the boxes to show the extent of the rest of the data. Outliers are data with values beyond the ends of the whiskers. By visually inspecting the boxplots in Figure 4 there seems to be a tendency that the celeriac models having MSE: $(5.88,4.77)$ respectively, generally have a better prediction ability than the carrot models having MSE: $(17.31,13.88)$. It also seems that there is a difference between some of the groups in each of the four models, which generally increases slightly in the beginning and then flattens out towards the end. Specifically for the carrots it seems that after day 4, the predictions starts to oscillate, as if an equilibrium has been reached. The same is the case for the celeriac after day 8 .

Figure 5 shows the result of all pairwise two-sided t-tests between all days in each model. The t-tests test the $H_{0}$ hypothesis, that two groups can be assumed to come from the same population at the $5 \%$ level of significance. The

\begin{tabular}{|c|c|c|c|c|c|c|}
\hline & D2 & D4 & D8 & D10 & D12 & D14 \\
\hline D2 & 0 & 2 & 2 & 2 & 2 & 2 \\
\hline D4 & 2 & 0 & 0 & 0 & 2 & 1 \\
\hline D8 & 2 & 0 & 0 & 0 & 2 & 0 \\
\hline D10 & 2 & 0 & 0 & 0 & 2 & 1 \\
\hline D12 & 2 & 2 & 2 & 2 & 0 & 2 \\
\hline D14 & 2 & 1 & 0 & 1 & 2 & 0 \\
\hline
\end{tabular}

\begin{tabular}{|c|c|c|c|c|c|c|}
\hline & D2 & D4 & D8 & D10 & D12 & D14 \\
\hline D2 & 0 & 2 & 2 & 2 & 2 & 2 \\
\hline D4 & 2 & 0 & 1 & 2 & 2 & 2 \\
\hline D8 & 2 & 1 & 0 & 2 & 2 & 2 \\
\hline D10 & 2 & 2 & 2 & 0 & 1 & 1 \\
\hline D12 & 2 & 2 & 2 & 1 & 0 & 0 \\
\hline D14 & 2 & 2 & 2 & 1 & 0 & 0 \\
\hline
\end{tabular}

Figure 5. Both tables show all pairwise 2-sided t-tests between all groups of the carrot model(a) and the celeriac model(b). 0 indicates neither model can reject the $H_{0}$-hypothesis (i.e. we accept that the means are equal). 1 indicates one of the two models rejects the $H_{0}$-hypothesis, and 2 indicates both models reject the $H_{0}$-hypothesis(i.e. the groups are significantly different at a $5 \%$ level). Both tables are symmetrical respectively. The diagonal contain only zeros.

statistical tests show that for carrots we are able to verify a significant change in the mean from day 2 to day 4 . After day 4 we are not able to verify a significant change in mean for the carrots, which could indicate a steady state has been reached. However, for the celeriac we are able to significantly track a change from day to day until day 12 , with the exception of day 8 where some uncertainty appears.

As mentioned in the section about the experimental setup, the vegetables were kept in the refrigerator in plastic bags. 


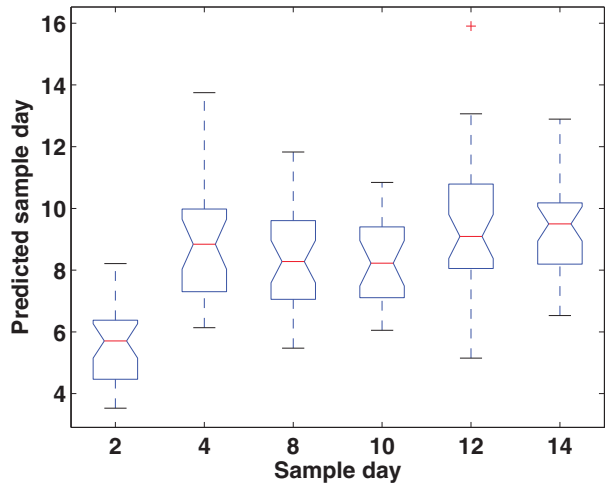

(a)

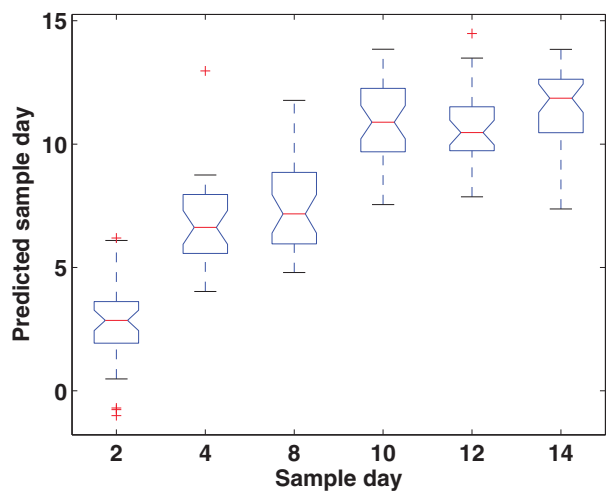

(c)

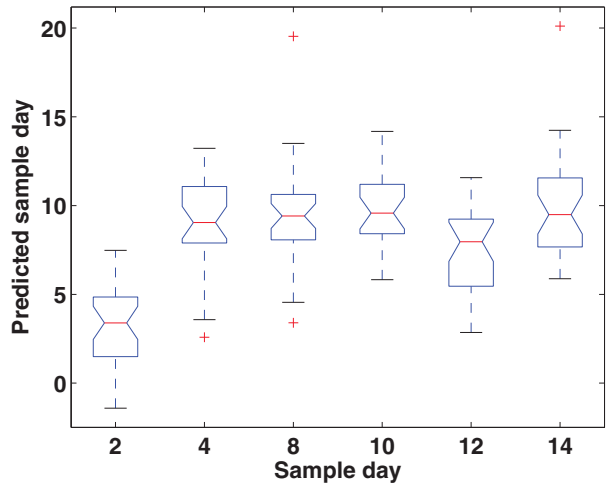

(b)

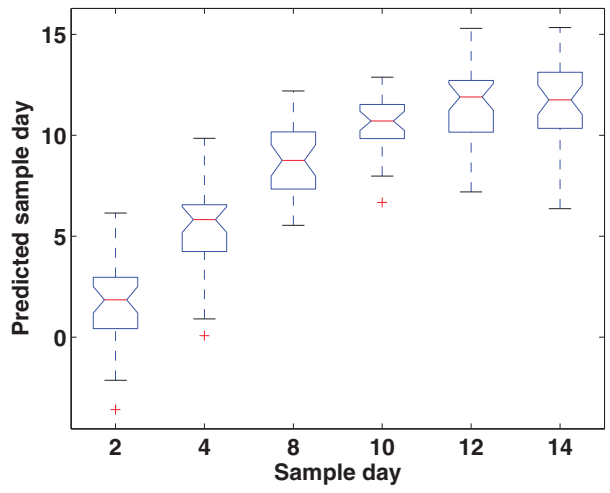

(d)

Figure 4. All figures show predictions grouped by true sample day. The top two plots show predictions for the carrot samples, while the bottom two plots show the predictions of the celeriac. The two leftmost figures show the model trained with LOO-CV on dataset A and tested on dataset B, while the rightmost figures show the model trained with LOO-CV on dataset B and tested on dataset A.

Plastic bags are not able to isolate oxygen molecules, which is why we believe the change in the spectra is caused by oxidation of the vegetables. An oxidation causes browning/graying of celeriac and carrots to become more pale. An increasing brown/gray color is a change in a wide range of the spectrum, and is essentially a change of brightness. The most significant components describing the celeriac consists of wavelengths from the entire visible spectrum, which coincides with a general shift in brightness. For the carrots the components seem to have a tendency to lie in the red/NIR area, which also coincides with a general more pale appearance, or removal of redness/orangeness, which essentially is an oxidation of the beta-carotene. This is exactly the color change to expect in an oxidation process of these vegetables.

\section{Conclusions}

An objective measure of the quality change of carrots and celeriac was proposed which uses multispectral image analysis. Six images were recorded over 14 days, for two different data sets. Each carrot or celeriac piece was isolated using a combination of a Canonical Discriminant Analysis and watershed algorithm, for a total of around 200 pieces per training and test set, for both carrots and celeriac pieces respectively. A set of 3249 features were extracted for each vegetable piece, giving rise to a very ill posed $p>>n$ problem. A special regression technique, Least Angle Regression-Elastic Net, was performed on both the carrot and celeriac data sets. Test and training were interchanged, resulting in two models per vegetable type. These were estimated to check the repeatability and statistical tests were performed to check if it in fact was possible to discriminate between the different days predicted on behalf of the estimated models.

The results showed that the celeriac predictions were somewhat better than the carrots, although a trend was seen in both. We see that there is a large change from day 2 to day 4 in the reflectance spectrum for both carrots and celeriac, and for the celeriac we see the change continuing until day 12. The pairwise two sided t-tests showed exactly that these changes were statistically significant at a $5 \%$ level of signif- 
icance. The corresponding sensory tests showed no difference over the 14 days, which makes it the more important that we are able to detect minor changes using multispectral imaging.

\section{Acknowledgements}

The authors would like to thank Rene Thrane and Peter Reimer Stubbe for carrying out the manual experiments in the laboratory at the National Food Institute of Denmark. This study was financially supported by The Danish Food Industry Agency

\section{References}

[1] Jens Adler-Nissen. The continuous wok - a new unit operation in industrial food processes. J. Food Process Engin., 25:435-453, 2002.

[2] Jens Adler-Nissen. Industrial stir frying. Asia Pacific Food Industry, 17(5):32-34, 2005.

[3] Jens Adler-Nissen. Continuous wok-frying of vegetables: Process parameters influencing scale up and product quality. Journal of Food Engineering, 83:5460, 2007.

[4] Huynh N. Duy Bao, Sigurjon Arason, and Kristin A. Porarinsdottir. Effects of dry ice and superchilling on quality and shelf life of arctic charr (salvelinus alpinus) fillets. International Journal of Food Engineering, 3(3):art.7, 2007.

[5] Maria G. Burgaard, A. Matzen, and Jens AdlerNissen. Kontinuerlig wok til industriel brug. Plus Proces, (6):24-26, 2004.

[6] Line H. Clemmesen, Michael E. Hansen, and Bjarne K. Ersbøll. A comparison of dimension reduction methods with application to multi-spectral images of sand used in concrete. Machine Vision and Applications, 2009.

[7] B. Efron, T. Hastie, I. Johnstone, and R. Tibshirani. Least angle regression. Annals of Statistics, 32(2):407-499, 2004.

[8] David D. Gomez, Line H. Clemmensen, Bjarne K. Ersbøll, and Jens M. Carstensen. Precise acquisition and unsupervised segmentation of multi-spectral images. Computer Vision and Image Understanding, 106(2-3):183-193, 2007.

[9] Rafael C. Gonzalez, Richard E. Woods, and Steven L. Eddins. Digital Image Processing. 2 edition, 2002.

[10] Per C. Hansen. Rank-Deficient and Discrete Ill-Posed Problems. SIAM, 1998.
[11] Trevor Hastie, Robert Tibshirani, and Jerome Friedman. Elements of Statistical Learning: data mining, inference and prediction. Springer-Verlag, 2001.

[12] A. E. Hoerl and R. W. Kennard. Ridge regression. biased estimation for nonorthogonal problems. Technometrics, 12(1):55-67, 1970.

[13] Nobuyuki Otsu. A threshold selection method from gray-level histograms. IEEE Transactions on Systems, Man, and Cybernetics, 9(1):62-66, 1979.

[14] Robert Tibshirani. Regression shrinkage and selection via the lasso. Journal of the Royal Statistical Society. Series B (Methodological), 58(1):267-288, 1996.

[15] Andrey N. Tikhonov. Solutions of incorrectly formulated problems and the regularization method. Soviet Mathematics Doklady, 4:1035-1038, 1963. English translation of Dokl. Akad. Nauk. SSSR, 151:501-504, 1963.

[16] Sonia Z. Vina and Alicia R. Chaves. Texture changes in fresh cut celery during refrigerated storage. Journal of the Science of Food and Agriculture, 83:1308-1314, 2003.

[17] Hui Zou and Trevor Hastie. Regularization and variable selection via the elastic net. Journal of the Royal Statistical Society, 67(2):301-320, 2005. 